JSURGRES-D-15-00611 (R1)

\title{
LIQUID PLASMA USE DURING "SUPER" MASSIVE TRANSFUSION PROTOCOL
}

\author{
Casey J Allen, Jonathan P Meizoso, Mena M Hanna, Jose L Mora, Juliet J Ray, Nicholas \\ Namias, Roman Dudaryk, and Kenneth G Proctor
}

Dewitt-Daughtry Departments of Surgery and Anaethesiology, Divisions of Trauma and Critical Care. University of Miami Miller School of Medicine, Miami, FL 33136, USA

CJA is directly responsible for all aspects of this study. He participated in the collection, analysis and interpretation of data; drafting and revision of the manuscript, figures and tables. JPM, $\mathrm{MMH}, \mathrm{JM}$ and JJR participated in the revision of the manuscript, figures and tables. NN and RD were medically responsible for the patients and participated in the review and revision of the manuscript, figures, and tables. KGP had overall responsibility for the study; including conception and experimental design; analysis and interpretation of data; drafting and revision of the manuscript, figures and tables; statistical expertise and evaluation; obtaining funding for this project; supervision.

Supported in part by: Grants \#N140610670 from the Office of Naval Research and \#09078015 from U.S. Army Medical Research \& Materiel Command

Portions of these data were presented at the 2015 Academic Surgical Congress, Las Vegas, NV, February 8-10, 2015.

None of the authors have declared conflicts of interest.

Address for manuscript correspondence:

Kenneth G. Proctor, Ph.D.

Professor of Surgery

Divisions of Trauma and Surgical Critical Care

Daughtry Family Department of Surgery

University of Miami School of Medicine, Ryder Trauma Center

1800 NW 10th Ave. Miami, FL 33136

305-585-1178 (office), 305-326-7065 (fax), kproctor@miami.edu

SHORT TITLE: LP and "Super" MTP

KEYWORDS: acute coagulopathy of trauma; FFP; hemostatic resuscitation; MTP 


\section{ABSTRACT}

Background: A massive transfusion protocol (MTP) presents a logistical challenge for most blood banks and trauma centers. We compare the ratio of packed red blood cells (PRBC):plasma transfused over serial time points in those requiring MTP (10-30 units PRBC/24hrs) to those requiring “super” MTP (S-MTP; >30 units PRBC/24hrs), and test the hypothesis that changes in allocation of blood products with use of readily transfusable liquid plasma (LP) improves the ratio of PRBC:plasma during S-MTP.

Materials and Methods: All transfused trauma patients $(n=1,305)$ from $1 / 09$ to $3 / 15$ were reviewed. PRBC:plasma was compared for MTP $(n=277)$ and S-MTP $(n=61)$ patients, before and after the availability of LP at our institution. Data are reported as mean \pm standard deviation or median(interquartile range).

Results: Age was 41 $\pm 19 y$, 52\% blunt mechanism, injury severity score $32 \pm 16$, and $46.3 \%$ mortality. In $24 \mathrm{hr}$, requirements were 17(14)units PRBC, 10(11)units plasma, with a PRBC:plasma of 1.6(0.8). Within the first hour, PRBC:plasma for S-MTP vs MTP was 2.1:1 vs 1.7:1 ( $\mathrm{p}=0.017)$. With LP, S-MTP patients received significantly lower PRBC:plasma at the first hour $(\mathrm{p}<0.001)$. Before institutional changes, PRBC:plasma positively correlated with PRBC transfused at hour $1\left(\mathrm{r}=0.410, \mathrm{R}^{2}=0.168, \mathrm{p}<0.001\right)$; after institutional changes and the advent of LP there was no correlation $\left(\mathrm{r}=0.177, \mathrm{R}^{2}=0.031, \mathrm{p}=0.219\right)$.

Conclusion: Within the first hour of transfusion, units of PRBC transfused positively correlated with PRBC:plasma ratios, and patients receiving S-MTP had higher PRBC:plasma ratios than those receiving MTP. Changes in our institution's MTP protocol to include LP improved the early ratio of PRBC:plasma transfused in patients requiring S-MTP.

\section{Level III Evidence}




\section{INTRODUCTION}

Hemorrhagic shock remains the leading cause of death in trauma patients aged 1 to 44 years old $[1,2]$. A massive transfusion protocol (MTP) is activated in many level 1 trauma centers when a patient presents with uncontrolled, life-threatening bleeding [3]. During MTP, the goal is to transfuse packed red blood cells (PRBC), plasma, and platelets in a similar ratio to that of whole blood; this minimizes dilutional coagulopathy and hypovolemia, and improves hemostatic resuscitation and subsequent outcomes [4-9]. Most recently, the Pragmatic, Randomized Optimal Platelet and Plasma Ratios (PROPPR) trial showed evidence of benefit of an early 1:1:1 transfusion [10]. Along with recent recommendations by the US Food and Drug Administration (FDA) including a 3-hour end point for studies focusing on traumatic hemorrhage [11], it is clear the goal therapy for hemostatic resuscitation is an immediate and balanced blood component delivery. The American College of Surgeons Trauma Quality Improvement Project issued guidelines for trauma resuscitation in November 2013, recommending that "Universal donor products should be immediately available on patient arrival to support ratio-based transfusion," and "If MTP triggers are met, transfuse universal plasma and $\mathrm{RBC}$ in a ratio between 1:1 and 1:2" [12]. These new guidelines represent a major shift in the paradigm of trauma resuscitation and blood product provision, and are likely to become accreditation requirements in the near future.

Proper implementation of an MTP depends on a 24/7/365 state of readiness in the blood bank for rapid delivery of components to the bedside. Practically, it may be difficult to continuously maintain a desired ratio of transfused components at least during active bleeding and ongoing resuscitation [13]. It is not unusual at urban trauma centers for an occasional patient to require 
"super" massive transfusion protocol (S-MTP; $>30$ units PRBC in $24 \mathrm{hr}$ ), which poses an extraordinary logistical challenge for most blood banks and trauma centers. The term "super" MTP is not a generally recognized classification, but it has been used in few reports [14, 15], to represent that fraction of the population that requires an exorbitant amount of blood products. Our institution has recently changed its blood product delivery method during MTP activations to make more plasma capable of immediate transfusion (thawed fresh frozen plasma [FFP] or liquid [never thawed] plasma [LP]) in higher quantities; see Table 1.

The purpose of this study was to compare the ratio of PRBC:plasma transfused over serial time points immediately following transfusion initiation in those requiring MTP vs S-MTP. We test the hypothesis that changes in the availability of readily transfusable plasma, made possible with LP, improves the ratio of PRBC:plasma transfused in patients requiring S-MTP.

\section{MATERIALS AND METHODS}

With the approval of the Institutional Review Board of the University of Miami Miller School of Medicine (Miami, FL) and Jackson Memorial Hospital, and waiver of informed consent, we reviewed all patients requiring blood component transfusions within six hours of arrival to the Ryder Trauma Center from 1/1/09 to 3/4/15.

Demographics, injury patterns, procedures, total fluid and transfusion requirements, and outcomes were analyzed in those who received $>10$ units PRBCS or plasma over $24 \mathrm{hr}$. Each component was recorded at serial time increments during MTP (10-30 units PRBC and S-MTP (>30 units PRBC). The institutional MTP policy and procedure changes on July 1, 2014 are 
detailed in Table 1.

To match cohorts of each era, propensity scores were assigned for each patient based on a logistic regression model using patient and injury characteristics (i.e., age, injury severity score [ISS], systolic blood pressure [SBP], heart rate [HR], Glasgow Coma Score [GCS], base excess [BE], hematocrit $[\mathrm{Hct}])$. A 1:1 fixed ratio nearest neighbor matching was performed to compare mortality between the cohorts to minimize bias without sacrificing power [16].

Statistical analyses were performed using SPSS version 22 (IBM Corporation; Armonk, NY). Parametric data are reported as mean \pm standard deviation and non-parametric are reported as median (interquartile range). Data were compared using a t-test or Mann Whitney U test, as appropriate. Categorical variables were compared using a chi-square or Fisher's exact test, as appropriate. Pearson correlation coefficient (r) tests were performed to detect correlations between units PRBC transfused and PRBC:plasma ratios. Statistical significance was determined at alpha level 0.05 .

\section{RESULTS}

A total of 1,305 patients were transfused, with 277 MTP and 61 S-MTP. For a more complete description, Table 2 details the overall population. In $24 \mathrm{hr}$, transfusion requirements were 17(14)units of PRBC and 10(11) units of plasma, with a PRBC:plasma of 1.6(0.8). In comparing population differences between those who received MTP versus S-MTP, Table 3 shows how those who receive S-MTP are inherently more severely injured, require more fluids and blood products, and have a significantly higher rate of mortality. 
To identify how blood component ratios differed according to time between these populations, the PRBC:plasma ratio for each patient was calculated at serial time increments and median values were plotted in Figure 1. Notably, within the first hour, S-MTP patients received a higher PRBC:plasma ratio of $2.1: 1$ vs 1.7:1 in MTP patients $(\mathrm{p}=0.017)$.

When comparing the patient populations before and after the institutional protocol changes, Table 4 shows that patient demographics were similar yet ISS $(31 \pm 16$ vs $46 \pm 19, p<0.001)$ and mortality $(43.8 \%$ vs $59.3 \%, \mathrm{p}=0.038)$ were significantly different. As the difference in injury severity could account for the differences in mortality, we used a 1:1 fixed ratio nearest neighbor propensity score match. With this, 62 patients with similar patient characteristics (age, ISS, BE, SBP, HR, Hct) were compared and there was no difference in mortality $(54.8 \%$ vs $58.1 \%$, $\mathrm{p}=1.000)$.

In assessing the effectiveness of institutional changes, Figure 2 shows the PRBC:plasma ratio during MTP and S-MTP at serial time points. S-MTP patients received significantly lower PRBC:plasma ratios within the first two hours following initial transfusion (all $\mathrm{p}<0.05$ ) following the advent of LP use.

To assess the change in PRBC:plasma ratio as a function of total blood requirements, Figure 3 shows that, in all patients, PRBC:plasma positively correlated with units PRBC transfused at hour $1\left(\mathrm{r}=0.343, \mathrm{R}^{2}=0.118, \mathrm{p}<0.001\right)$. Before institutional changes, units $\mathrm{PRBC}$ transfused 
positively correlated with PRBC:plasma at hour $1\left(\mathrm{r}=0.410, \mathrm{R}^{2}=0.168, \mathrm{p}<0.001\right)$, but not after the advent of LP ( $\left.r=0.177, \mathrm{R}^{2}=0.031, \mathrm{p}=0.219\right)$.

\section{DISCUSSION}

There are three major findings of this study: (1) PRBC:plasma positively correlated with units PRBC transfused within the first hour immediately following initial transfusion during MTP, (2) PRBC:plasma was higher in S-MTP vs MTP, and (3) the changes in our institution's availability of readily transfusable plasma improved early ratio of PRBC:plasma in patients requiring SMTP. These results support the conclusion that early resuscitation during MTP is improved by making more plasma readily available for immediate transfusion and adapting protocols to encompass the most efficient delivery system of blood products.

In trauma, the "golden hour" is critical to survival [17]. Only 3-5\% of all trauma patients require MTP but it is important to quickly identify these patients [3, 5, 18]. A PRBC:plasma ratio between 1:1 and 2:1 during initial transfusion has a survival benefit during MTP [19-21]. Duchesne et al. reported that survival was improved with PRBC:plasma of 1:1 vs 4:1 [19]. Sperry et al. reported lower $48 \mathrm{hr}$ mortality rates with a ratio of FFP:PRBC $\geq 1: 1.5$ [21]. A ratio of PRBC:plasma:platelets of at least 1:1:1 is associated with reduced mortality $[9,22,23]$. The recent evidence of benefit of a 1:1:1 transfusion was also found in the PROPPR trial [10]. The early availability of blood products within minutes of arrival using a transfusion ratio of 1:1:1 was associated with more patients achieving hemostasis and decreased hemorrhage-related deaths [10]. Based on recommendations from the FDA [11] and the guidelines set by the ACS [12], it is clear the goal therapy for hemostatic resuscitation is an immediate and balanced blood 
component delivery. Unfortunately, as our results show, it is not always possible to deliver the desired ratio during most extreme conditions. Logistical improvements on our MTP seem to solve this problem of the delivery of immediate and balanced blood components.

It is important to note that the goal ratios were between 1:1 and 2:1 during the study period, and the changes in MTP were made to improve allocation to facilitate that delivery. Those with MTP alone (10-30 units PRBCs) did not experience deficits in delivery prior to institutional changes (see Figure 1). When assessing the effects of the protocol changes (and LP) on regular MTP, there was no significant difference in delivery but it was still within the goal of $1: 1$ to $2: 1$ at all time points (see Figure 2A). Still, to this point, Figure 3 shows that, regardless of MTP or SMTP, there was a correlation of increased PRBC:plasma ratio with total blood requirements, and this was not seen after institutional changes.

There are no studies assessing blood component ratios with $>30$ units PRBC during MTP. Figure 2 showed that there was a lag phase in the first hours in those requiring S-MTP suggesting the need for more immediately available plasma. The inability to maintain the target ratio in S-MTP may be complicated by multiple factors.

Plasma is ideally given with the first unit of PRBC $[24,25]$. Whereas PRBCs are stored in a refrigerator, plasma is stored frozen. It must be thawed in a water bath over 20 to 30 min [26], which can delay its administration during MTP [27]. Waiting for FFP to thaw makes this concurrent administration of blood components difficult to maintain the desired 1:1 to 2:1 ratio throughout the critical resuscitation period. After $24 \mathrm{hr}$, FFP is relabeled as "thawed plasma" and 
can be stored and/or utilized for four more days [28]. This product is immediately transfusable, but supply may be limited because of shelf-life. Another concern is the limited donor pool of AB type FFP - only $4 \%$ of the population of the United States. There has been an increasing push for the use of LP over thawed plasma due to these logistical issues [13, 29]. With LP, there is no delay waiting for a thaw. This product can be available in higher quantities as it can be stored for up to 26 days [28, 30], which can preserve the supply of AB FFP.

The PROPPR trial was potentially limited by the low number of units of plasma available from blood suppliers [31]. Additionally, there was concern that there would be excessive wastage if plasma was routinely thawed in advance [31]. Several centers experienced significant wastage due to their need to have more plasma available (thawed and discarded) for the study protocol; yet the single institution which used LP (Houston) experienced one of the lowest wastage rates of $0.4 \%$ [31]. This utilization improvement may be even more pronounced in smaller hospitals with less volume of patients requiring MTP. As plasma use and turnover is likely less in these hospitals, we can speculate that they may benefit more from the wastage advantage LP offers.

Trauma centers have variable blood product allocation strategies for MTP $[3,5,7,8]$ and very few institutions use LP at their hospitals [31]. With the advent of LP, our institution changed its MTP policy; see Table 1. Jackson Memorial Hospital's "Policies and Procedures: Massive Transfusion Protocol" changes took effect on 6/1/14. Prior to that date, five units of uncrossmatched PRBC and two units of group AB plasma were available until a type-andcrossmatch was obtained and type-specific FFP was thawed and delivered. Upon MTP activation, blood components were delivered via the TransLogic $₫$ Pneumatic Tube System from 
the blood-bank to the trauma resuscitation unit. With the new policy, eight units of universal donor, uncrossmatched $\mathrm{PRBC}$ and eight units of readily transfusable plasma (thawed AB plasma and LP) are immediately available. All blood products are now transported in a cooler by a laboratory "runner" with instructions to stay one round/batch ahead for the duration of the MTP activation. Prior to this protocol, there was still blood immediately available for massive transfusion. Therefore, the new protocol would not have any effect on time until first unit transfused, regardless of PRBC or plasma component. The changes were made to address the availability of readily transfusable plasma in order to keep up with the $1: 1$ to $2: 1$ ratio required by the American College of Surgeons. As time-to-transfusion may vary depending on patient situation, the PRBC:plasma ratio should be maintained 1:1 to 2:1. Therefore, this is the end-point assessed in our report.

With this institutional change, the early PRBC:plasma ratio in patients requiring S-MTP improved toward the 1:1 target. It is difficult to determine whether this improvement is due to LP, to the change in blood products allocation methodology, or to a combination of the two factors. To be clear, no unit of plasma is specifically designated as LP or thawed FFP during a MTP. LP has been added to our existing blood bank to have readily available plasma for transfusion. Our blood bank still uses FFP as it has a longer shelf life; most patients can wait the 20-30 min for it to thaw. Sometimes, FFP may be thawed but not transfused for one patient, and then must be used within 5 days. Depending on the situation, there may be an abundance of thawed FFP readily available for MTP, and these would be the first units allocated. However, in times of exorbitant blood requirements (S-MTP), this supply might not be enough to keep up with the $1: 1$ to $2: 1$ required, thus LP is available. In another scenario, the blood bank may not 
have much recently thawed FFP for MTP use, thus LP would be employed much earlier following protocol activation. Ultimately, even if LP is not directly used in each MTP, its availability is what facilitates our new capability to balance blood product ratios.

We cannot explain why the mortality rate of those requiring MTP was higher after our institutional changes, however any apparent difference in outcomes is probably confounded by the small sample size and by differences in injury severity. No attempt was made to measure the effects of ratios on coagulation function; hence, little can be ascertained about the effects of liquid plasma. Also, the results of our propensity score match to control for these patient and injury differences showed no difference in mortality between eras. However this was not the goal of this study. It is already established that a PRBC:plasma ratio between $1: 1$ and 2:1 has an added survival benefit to patients requiring MTP [19-21]; with most recently 1:1 being the gold standard [10]. The purpose of this study was not to assess if these changes directly provide a mortality benefit, rather if LP facilitates the delivery of this target ratio that is becoming a guideline set by both the FDA and the ACS.

This is one of the first reports on the use of LP during MTP [29, 32], and an obvious limitation is the small number that received MTP after institutional changes. The fact is that this sample may not represent the entire trauma population. However, the differences in blood product administration were significant. The term "super" MTP is not a generally recognized classification, but the term has been used in few reports, with the definition varying $(>25-40$ units PRBCs) $[14,15]$. For the purposes of this study, we identified those that required the top $20 \%$ of total blood component requirements amongst all patients who receive MTP and 
arbitrarily set the value at $>30$ units. Another limitation is that this is a single institution review of observational data without randomization, and therefore baseline differences between groups could not be directly controlled. Aside from the MTP, resuscitative and operative strategies were based upon physician discretion without a set protocol. However, our group of trauma surgeons and anesthesiologists follow current guidelines and resuscitate to similar hemodynamic parameters. The timing of blood products transfused was according to first unit of PRBC given. As our institution has uncrossmatched, universal donor PRBCs immediately available for all trauma patients, it is likely these patients were initially transfused units not a part of the MTP. Therefore, initial ratios may be skewed towards a higher PRBC component, but this bias would have been imposed equally on all subgroups. Another limitation is that we did not directly measure the effect of LP but rather the logistical benefits it offers. Finally, the purpose of this study was not to determine optimal blood component ratios to improve patient outcomes. This study was based on the assumption that a balanced ratio of blood components is the goal during MTP.

\section{CONCLUSIONS}

In summary, within the first hour immediately following initial transfusion during MTP, (1) PRBC:plasma ratios positively correlated with units PRBC transfused, (2) patients receiving >30units PRBC in 24hrs (S-MTP) had significantly higher PRBC:plasma transfusion ratios than those of MTP, and (3) the improvements in allocation of blood products with use of readily transfusable plasma (enabled with use of LP) improve the ratio of PRBC:plasma transfused within the first hours in patients requiring S-MTP. Making more plasma readily available for immediate transfusion and adapting protocols to encompass the most efficient delivery system of 
blood products from the blood-bank to the resuscitation unit improves the process of early resuscitation during MTP.

\section{ACKNOWLEDGEMENT}

We recognize the nursing and administrative staff at Ryder Trauma Center for their cooperation and assistance with the patients and their families.

\section{REFERENCES}

[1] Holcomb JB, Pati S. Optimal Trauma Resuscitation with Plasma as the Primary Resuscitative Fluid: The Surgeon's Perspective. Hematology. 2013:656-59.

[2] Kauvar DS, Wade CE: The epidemiology and modern management of traumatic hemorrhage: US and international perspectives. Crit Care. 2005 9(Supple 5):S1-S9.

[3] Schuster KM, Davis KA, Lui FY, Maerz LL, Kaplan LJ. The status of massive transfusion protocols in United States trauma centers: massive transfusion or massive confusion? Transfusion. 2010 Jul;50(7):1545-51.

[4] Smith CE, Bauer AM, Pivalizza EG, Tanaka K, Boral L, Shander A, Waters JH. Massive transfusion protocol (MTP) for hemorrhagic shock. American Society of Anesthesiologists Committee on Blood Management. Accessed 12/15/2014

[5] Nunez TC, Young PP, Holcomb JB, Cotton BA. Creation, Implementation, and Maturation of a Massive Transfusion Protocol for the Exsanguinating Trauma Patient. J Trauma. Jun 2010; 68(6): 1498-1505.

[6] Borgman MA, Spinella PC, Perkins JG, Grathwohl KW, Repine T, Beekley AC, Sebesta J, Jenkins D, Wade CE, Holcomb JB. The ratio of blood products transfused affects mortality 
in patients receiving massive transfusions at a combat support hospital. J Trauma. 2007;63(4):805-813.

[7] Riskin DJ, Tsai TC, Riskin L, Hernandez-Boussard T, Purtill M, Maggio PM, Spain DA, Brundage SI. Massive transfusion protocols: the role of aggressive resuscitation versus product ratio in mortality reduction. J Am Coll Surg. 2009 Aug;209(2):198-205.

[8] O'Keeffe T, Refaai M, Tchorz K, Forestner JE, Sarode R. A massive transfusion protocol to decrease blood component use and costs. Arch Surg. 2008; 143:686-690, discussion 690-691.

[9] Khan S, Allard S, Weaver A, Barber C, Davenport R, Brohi K. A major hemorrhage protocol improves the delivery of blood component therapy and reduces waste in trauma massive transfusion. Injury. 2013 May; 44(5); 587-92.

[10] Holcomb JB, Tilley BC, Baraniuk S, Fox EE, Wade CE, Podbielski JM, del Junco DJ, Brasel KJ, Bulger EM, Callcut RA, et al. Transfusion of plasma, platelets, and red blood cells in a $1: 1: 1$ vs a $1: 1: 2$ ratio and mortality in patients with severe trauma: the PROPPR randomized clinical trial. JAMA. 2015 Feb 3;313(5):471-82. doi: 10.1001/jama.2015.12.

[11] US Food and Drug Administration. Product development program for interventions in patients with severe bleeding due to trauma or other causes, 2010. Available at http://www.fda.gov/BiologicsBloodVaccines/NewsEvents/WorkshopsMeetingsConference s/ucm241913.htm. Accessed March 1, 2015.

[12] American College of Surgeons. Quality improvement project: trauma resuscitation best practice guidelines. Chicago (IL): American College of Surgeons; 2013.

[13] Goodnough LT, Spain DA, Maggio P. Logistics of transfusion support for patients with massive hemorrhage. Curr Opin Anaesthesiol. 2013 Apr;26(2):208-14. 
[14] Brohi K, Mark K, Dutton R. Recombinant Factor VIIa (NovoSeven) for Traumatic Coagulopathy. Trauma.org. Accessed January 13, 2015. Available at http://www.trauma.org/archive/resus/FactorVIIa.html.

[15] Treatment of Suspected Invasive Fungal Infection in War Wounds. Accessed January 13, 2015. Available at http://www.usaisr.amedd.army.mil/assets/cpgs/Invasive_Fungal_Infection_in_War_Woun ds 1 Nov 12.pdf.

[16] Austin PC. Statistical criteria for selecting the optimal number of untreated subjects matched to each treated subject when using many-to-one matching on the propensity score. Am J Epidemiol. 2010;172(9):1092-1097.

[17] Crowley RA. A total emergency medical system for the state of Maryland. Md State Med J, 45(19754), pp. 37-45.

[18] Bhananker SM, Ramaiah R. Trends in trauma transfusion. Int J Crit Illn Inj Sci. 2011 JanJun; 1(1): 51-56.

[19] Duchesne JC, Hunt JP, Wahl G, Marr AB, Wang YZ, Weintraub SE, Wright MJ, McSwain NE Jr. Review of current blood transfusions strategies in a mature level I trauma center: were we wrong for the last 60 years? J Trauma. 2008;65:272-276. discussion 276-278.

[20] Holcomb JB, Wade CE, Michalek JE, Chisholm GB, Zarzabal LA, Schreiber MA, Gonzalez EA, Pomper GJ, Perkins JG, Spinella PC, et al. Increased plasma and platelet to red blood cell ratios improves outcome in 466 massively transfused civilian trauma patients. Ann Surg. $2008 ; 248: 447-458$.

[21] Sperry JL, Ochoa JB, Gunn SR, Alarcon LH, Minei JP, Cus chieri J, Rosengart MR, Maier RV, Billiar TR, Peitzman AB, et al. An FFP:PRBC transfusion ratio $\geq 1: 1.5$ is 
associated with a lower risk of mortality after massive transfusion. J Trauma. 2008;65:986993.

[22] Vaslef, SN, Knudsen NW, Neligan PJ, Sebastian MW. Massive Transfusion Exceeding 50 Units of Blood Products in Trauma Patients. J Trauma. 2002 Aug;53(2):291-5.

[23] Holcomb JB, del Junco DJ, Fox EE, Wade CE, Cohen MJ, Schreiber MA, Alarcon LH, Bai Y, Brasel KJ, Bulger EM, et al. The Prospective, Observational, Multicenter, Major Trauma Transfusion (PROMMTT) Study: comparative effectiveness of a time-varying treatment with competing risks. JAMA Surg. 2013 Feb; 148(2):127-36.

[24] Teixeria PG, Inaba K, Shulman I, Salim A, Demetriades D, Brown C, Browder T, Green D, Rhee P. Impact of plasma transfusion in massively transfused trauma patients. J Trauma. 2009; 66:693-697.

[25] Zink KA, Sambasivan CN, Holcomb JB, Chisholm G, Schreiber MA. A high ratio of plasma and platelets to packed red blood cells in the first 6 hours of massive transfusion improves outcomes in a large multicenter study. Am J Surg. 2009. 197:565-570.

[26] O'Shaughnessy DF, Atterbury C, Bolton-Maggs P, Murphy M, Thomas D, Yates S Williamson LM, British Committee for Standards in Haematology, Blood Transfusion Task Force: Guidelines for the use of fresh-frozen plasma, cryoprecipitate and cryosupernatant. $\mathrm{Br}$ J Haematol. 2004, 126: 11-28.

[27] Mehr CR, Gupta R, von Recklinghausen FM, Szczepiorkowski ZM, Dunbar NM. Balancing risk and benefit: Maintenance of a thawed group A plasma inventory for trauma patients requiring massive transfusion. J Trauma Acute Care Surg. 2013 Jun;74(6):1425-31.

[28] Deloughery, T. Logistics of Massive Transfusions. Hematology. 2010: 1; 470-473. 
[29] Johansson PI, Stensballe J, Oliveri R, Wade CE, Ostrowski SR, Holcomb JB. How I treat patients with massive hemorrhage. Blood. 2014 Nov 13;124(20):3052-8.

[30] Matijevic N, Wang YW, Cotton BA, Hartwell E, Barbaeu JM, Wade CE, Holcomb JB. Better hemostatic profiles of never-frozen liquid plasma compared with thawed fresh frozen plasma. J Trauma Acute Care Surg. 2013;74(1):84-90; discussion 90-81.

[31] Novak DJ, Bai Y, Cooke RK, Marques MB, Fontaine MJ, Gottschall JL, Carey PM, Scanlan RM, Fiebig EW, Shulman IA, et al. Making thawed universal donor plasma available rapidly for massively bleeding trauma patients: experience from the Pragmatic, Randomized Optimal Platelets and Plasma Ratios (PROPPR) trial. Transfusion. 2015 Mar 30. doi: 10.1111/trf.13098. [Epub ahead of print]. PMID: 25823522.

[32] Dudaryk R, Hess AS, Varon AJ, Hess JR. What is new in the blood bank for trauma resuscitation. Curr Opin Anaesthesiol. 2015 Apr;28(2):206-9.

\section{TABLES/FIGURES}

Table 1: Policy and Procedural changes at Ryder Trauma Center, massive transfusion protocol (MTP).

* 4-round cycle, repeated as needed.

Round-1: 5 units PRBC, 5 units plasma.

Round-2: 5 units PRBC, 5 units plasma, 10 units platelets.

Round-3: 5 units PRBC, 5 units plasma.

Round-4: 5 units PRBC, 5 units plasma, 10 units platelets, 10 units cryoprecipitate.

Table 2: Characteristics of all patients who receive MTP $(\mathbf{n = 3 3 8 )}$. Data are reported as mean \pm standard deviation and median (interquartile range).

Table 3: Comparison of patients who receive MTP (10-29 total units PRBCS) to those of SMTP ( $\geq \mathbf{3 0}$ units PRBCs) (n=338). Data are reported as mean \pm standard deviation and median (interquartile range).

\section{Figure 1: Ratio of PRBC:Plasma Administered at serial time points, MTP vs SMTP} $(\mathbf{n = 3 3 8 )}$. Data points are reported as median values for the population. Data were compared using a Mann Whitney U test. *Denotes statistical significance of $\mathrm{p}<0.05$. 
Table 4: Comparison of patients who receive any MTP before and after institutional change $(\mathbf{n}=\mathbf{3 3 8})$. Data are reported as mean \pm standard deviation and median (interquartile range).

Figure 2A: Ratio of PRBC:Plasma Administered during MTP at serial time points, before and after institutional change $(\mathbf{n}=\mathbf{2 7 7})$. Data points are reported as median values for the population. Data were compared using a Mann Whitney U test. *Denotes statistical significance of $\mathrm{p}<0.05$.

Figure 2B: Ratio of PRBC:Plasma Administered during S-MTP at serial time points, before and after institutional change $(\mathbf{n}=\mathbf{6 1})$. Data points are reported as median values for the population. Data were compared using a Mann Whitney U test. *Denotes statistical significance of $p<0.05$.

Figure 3: Correlations between units PRBC transfused and PRBC:plasma ratios, before and after institutional changes $(n=338)$. 
Table 1: Policy and Procedural changes at Ryder Trauma Center, massive transfusion protocol (MTP).

\begin{tabular}{|c|c|}
\hline Pre-institutional change (before July 1, 2014) & Post-institutional change (after July 1, 2014) \\
\hline $\begin{array}{l}\text { Two (2) units of group AB FFP available } \\
\text { immediately until type and crossmatch obtained }\end{array}$ & $\begin{array}{l}\text { Eight (8) units of thawed group } \mathrm{AB} \text { plasma or } \\
\text { liquid plasma (LP) available immediately until } \\
\text { type and crossmatch obtained }\end{array}$ \\
\hline $\begin{array}{l}\text { Five (5) units universal donor, uncrossmatched } \\
\text { PRBCs available immediately until type and } \\
\text { crossmatch obtained }\end{array}$ & $\begin{array}{l}\text { Eight }(8) \text { units universal donor, uncrossmatched } \\
\text { PRBCs available immediately until type and } \\
\text { crossmatch obtained }\end{array}$ \\
\hline $\begin{array}{l}\text { Blood components delivered via TransLogic } ® \\
\text { Pneumatic Tube System from blood-bank to } \\
\text { trauma resuscitation unit }\end{array}$ & $\begin{array}{l}\text { In the hands of a "runner", all blood products } \\
\text { transported via cooler with set PRBC:plasma ratio } \\
\text { of } 1: 1 \text { with addition of other product (platelet, } \\
\text { cryoprecipitate) as determined by batch number* }\end{array}$ \\
\hline $\begin{array}{l}\text { Additonal plasma for subsequent batches* } \\
\text { available upon thawing of FFP }\end{array}$ & $\begin{array}{l}\text { Additonal plasma for subsequent batches* } \\
\text { available upon thawing of FFP or with LP }\end{array}$ \\
\hline
\end{tabular}


Table 2: Characteristics of all patients who receive MTP $(n=338)$

\begin{tabular}{|llr|}
\hline Age, years & & $41 \pm 19$ \\
Gender & Male & $81 \%$ \\
& Female & $19 \%$ \\
Mechanism of Injury & Blunt & $52 \%$ \\
& Penetrating & $48 \%$ \\
SBP, mmHg & & $96 \pm 35$ \\
HR, bpm & & $108 \pm 31$ \\
GCS & $10 \pm 5$ \\
Base Excess, mEq/L & $-9 \pm 7$ \\
Hematocrit, \%) & $33 \pm 6$ \\
ISS & $33 \pm 17$ \\
Time to OR, min & $30(68)$ \\
24h crystalloids, mL & & $9605 \pm 5889$ \\
S-MTP & $18 \%$ \\
24h PRBC, units & & $17(14)$ \\
24h Plasma, units & & $10(11)$ \\
24h PRBC:Plasma & & $1.6(0.8)$ \\
LOS, days & $9(34)$ \\
Mortality & $46.3 \%$ \\
\hline
\end{tabular}

Parametric data are reported as mean \pm standard deviation and non-parametric are reported as median (interquartile range 
Table 3: Comparison of patients who receive MTP (10-29 total units PRBCS) to those of SMTP ( $\geq 30$ units PRBCs)

\begin{tabular}{|llrrr|}
\hline & & $\begin{array}{r}\text { MTP } \\
(\mathrm{n}=277)\end{array}$ & $\begin{array}{r}\text { S-MTP } \\
(\mathrm{n}=61)\end{array}$ & $\mathrm{p}$ value \\
\cline { 3 - 5 } Age, years & & $41 \pm 20$ & $37 \pm 16$ & 0.107 \\
Gender & Male & $81 \%$ & $77 \%$ & 0.477 \\
& Female & $19 \%$ & $23 \%$ & \\
Mechanism of Injury & Blunt & $53 \%$ & $46 \%$ & 0.396 \\
& Penetrating & $47 \%$ & $54 \%$ & \\
SBP, mmHg & & $97 \pm 35$ & $94 \pm 33$ & 0.564 \\
HR, bpm & & $106 \pm 32$ & $117 \pm 28$ & $\mathbf{0 . 0 1 8}$ \\
GCS & $10 \pm 5$ & $9 \pm 5$ & 0.524 \\
Base Excess, mEq/L & & $-8 \pm 7$ & $-9 \pm 7$ & 0.958 \\
Hematocrit, \% & & $33 \pm 6$ & $33 \pm 7$ & 0.576 \\
ISS & & $33 \pm 17$ & $34 \pm 16$ & 0.620 \\
Time to OR, min & $30(70)$ & $27(50)$ & 0.749 \\
24h crystalloids, mL & & $15(9)$ & $41(24)$ & $<\mathbf{0 . 0 0 1}$ \\
24h PRBC, units & $9024 \pm 5265$ & $12214 \pm 7649$ & $<\mathbf{0 . 0 0 1}$ \\
24h Plasma, units & & $31(14)$ & $<\mathbf{0 . 0 0 1}$ \\
24h PRBC:Plasma & $1.6(1.1)$ & $1.4(0.6)$ & $\mathbf{0 . 0 1 2}$ \\
LOS, days & $10(39)$ & $3(23)$ & 0.274 \\
Mortality & $42.3 \%$ & $63.9 \%$ & $\mathbf{0 . 0 0 3}$ \\
\hline
\end{tabular}

Parametric data are reported as mean \pm standard deviation and non-parametric are reported as median (interquartile range). 
Table 4: Comparison of patients who receive any MTP before and after institutional change $(n=338)$

\begin{tabular}{|llrrr|}
\hline & & $\begin{array}{r}\text { Pre-institutional } \\
\text { change }(\mathrm{n}=281)\end{array}$ & $\begin{array}{r}\text { Post-institutional } \\
\text { change }(\mathrm{n}=57)\end{array}$ & $\mathrm{p}$ value \\
\cline { 3 - 4 } Age, years & & $40 \pm 19$ & $44 \pm 19$ & 0.178 \\
Gender & Male & $79.7 \%$ & $84 \%$ & 0.582 \\
& Female & $20.3 \%$ & $16 \%$ & \\
Mechanism of Injury & Blunt & $52.3 \%$ & $47 \%$ & 0.562 \\
& Penetrating & $47.7 \%$ & $53 \%$ & \\
SBP, mmHg & & $97 \pm 34$ & $92 \pm 37$ & 0.334 \\
HR, bpm & $110 \pm 30$ & $102 \pm 34$ & 0.123 \\
GCS & $10 \pm 5$ & $9 \pm 5$ & 0.140 \\
Base Excess, mEq/L & $-9 \pm 7$ & $-8 \pm 5$ & 0.519 \\
Hematocrit, \% & & $33 \pm 6$ & $34 \pm 6$ & 0.229 \\
ISS & $31 \pm 16$ & $46 \pm 19$ & $<\mathbf{0 . 0 0 1}$ \\
S-MTP & $19 \%$ & $18 \%$ & 1.000 \\
24h PRBC, units & $17(14)$ & $16(10)$ & 0.860 \\
24h FFP, units & $10(11)$ & $10(6)$ & 0.543 \\
24h PRBC:FFP & $1.6(1.0)$ & $1.6(0.8)$ & 0.473 \\
Mortality & $43.8 \%$ & $59.3 \%$ & $\mathbf{0 . 0 3 8}$ \\
\hline
\end{tabular}

Parametric data are reported as mean \pm standard deviation and non-parametric are reported as median (interquartile range). 


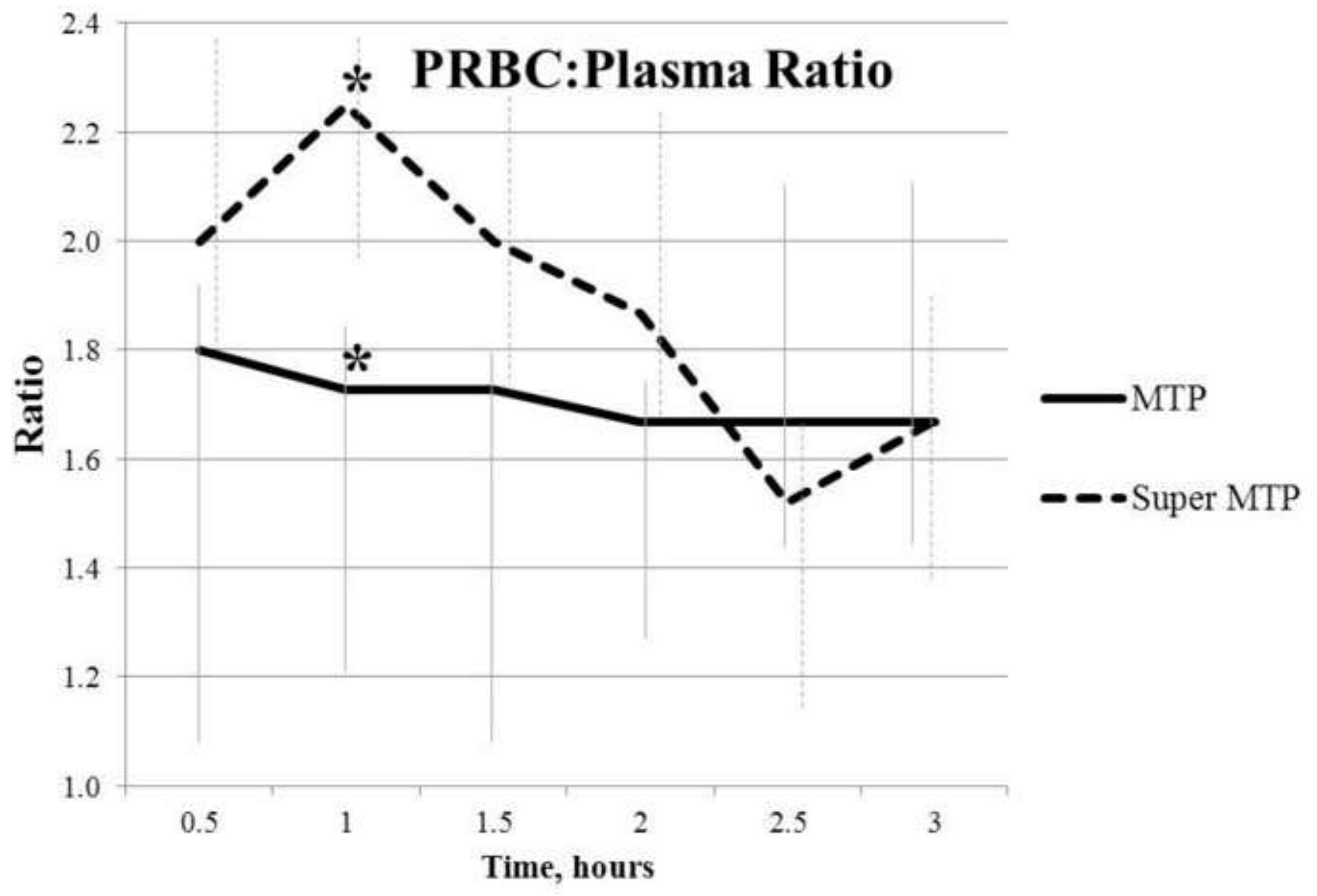



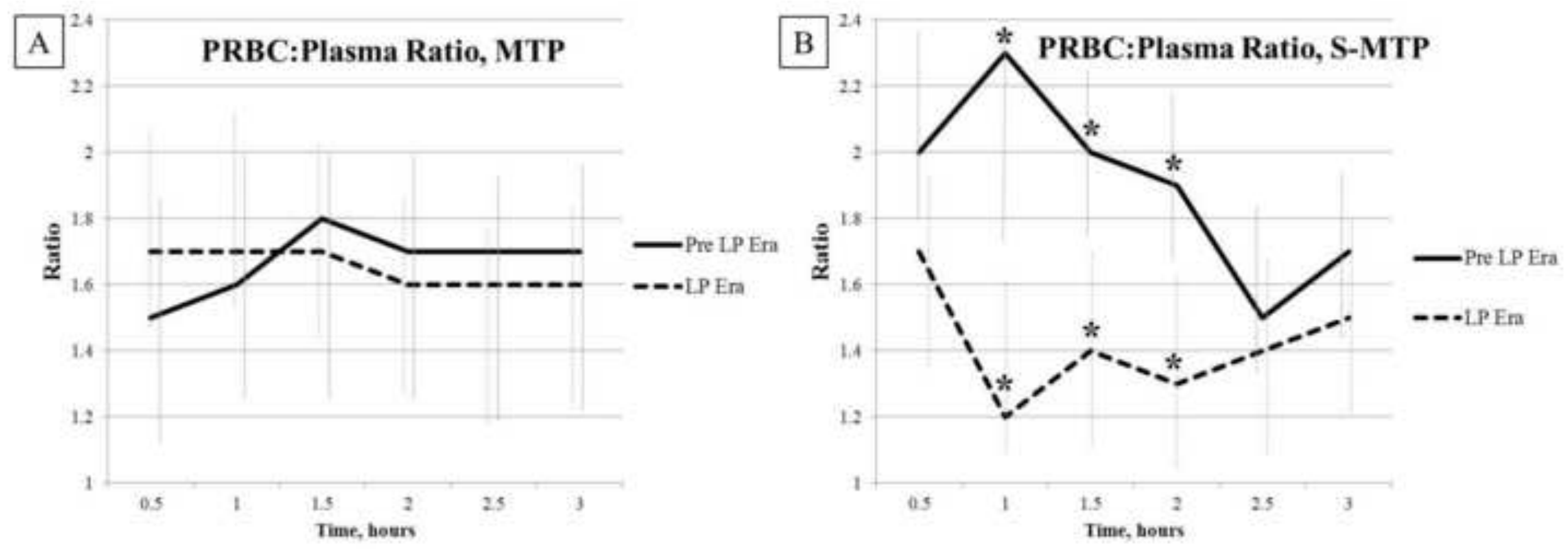

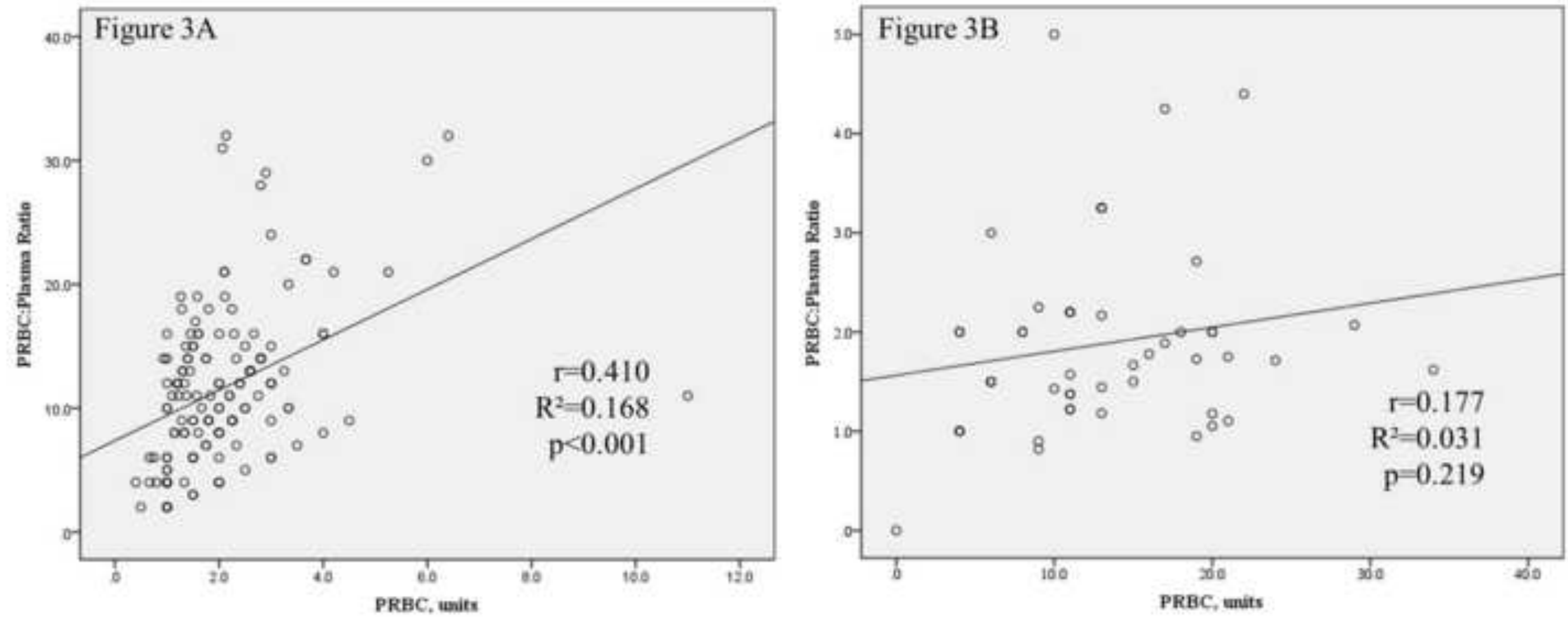

Figure 3A: Correlation between units PRBCs transfused and PRBC:Plasma ratio at hour 1, MTP before LP Era. n=281 Figure 3B: Correlation between units PRBCs transfused and PRBC:Plasma ratio at hour 1, MTP after LP Era. $n=57$ 